\title{
Introduction: Advances in intraoperative brain mapping
}

\author{
Richard W. Byrne, MD, ${ }^{1}$ Nader Sanai, MD, ${ }^{2}$ Jose A. Landeiro, MD, PhD, ${ }^{3}$ and Hugues Duffau, MD, PhD ${ }^{4}$
}

${ }^{1}$ Rush University Medical Center, Department of Neurosurgery, Chicago, Illinois; ${ }^{2 B}$ Barrow Neurological Institute, Neurological Surgery, Phoenix, Arizona; ${ }^{3}$ Centro de Ciências da Saúde, Faculdade de Medicina, Chefe do Serviço de Neurocirurgia do HUAP/UFF, Niteroi, Rio de Janeiro, Brazil; and ${ }^{4} \mathrm{Gui}$ de Chauliac, $\mathrm{CHU}$ de Montpellier, Neurosurgery, Montpellier, France

In the last decade, there has been significant interest among neurosurgeons in intraoperative brain mapping in cases of lesions in eloquent cortex and subcortical regions. This has occurred because foundational studies have provided strong evidence for the value of maximal safe resection of glioma. ${ }^{5,6}$ Further evidence of the important role of cortical and subcortical stimulation mapping in achieving a safe, maximal resection has come forth. ${ }^{2,3}$ Clinicians are also becoming aware of the limitations of preoperative imaging studies for definitive intraoperative decision-making. ${ }^{7}$

Preoperative mapping techniques are commonly used, but the extent to which they are relied upon is quite variable. ${ }^{7}$ In some centers, preoperative imaging techniques are used to guide the decision to choose cortical stimulation mapping, in other centers, it directs where the mapping may start, and in others, it is used in case the intraoperative mapping is ambiguous. It should be noted that there is significant variability in techniques in obtaining and processing this functional imaging data. Surgeons should become familiar both with this variability in general and with the protocols at their own center before relying on functional imaging for crucial decision-making.

In this issue of Neurosurgical Focus, we are pleased to show some of the recent advances and new applications of intraoperative brain mapping. A variety of techniques and protocols are discussed in this issue. A variety of pathologies, including glioma, metastatic tumor, vascular lesions, and epileptic foci, are addressed through transcortical, trans-sylvian, transventricular, and intrahemispheric routes. The common theme in each of these cases is to apply cortical and subcortical stimulation in at-risk areas in order to minimize risk and maximize resection. Readers should take note of stimulation parameters in each video and understand that there is practice variability that has evolved in each center.1,4 Whether a center is using monopolar or bipolar stimulation (or both), electrocorticography or not, common themes include careful observation and recording of stimulation response after escalation of intensity in a well-organized operative plan. Resection of the lesion is then performed, with the patient awake, or monitored, until the lesion is out or until the early onset of a new deficit. This careful monitoring is essential for safety and avoiding anything more than a temporary deficit. Important tips for comfortable and safe awake surgical techniques are also a common theme in the video cases in this issue.

Finally, emerging technologies and older technologies, such as transcranial magnetic stimulation and subdural grid mapping, are discussed, along with advantages and disadvantages.

This month's issue shows an excellent cross-section of the variety of contemporary applications of cortical stimulation mapping. We would like to thank the authors for their outstanding contributions to the field.

\section{References}

1. Corley JA, Nazari P, Rossi VJ, Kim NC, Fogg LF, Hoeppner TJ, et al: Cortical stimulation mapping parameters for functional mapping. Seizure 45:36-41, 2017

2. De Witt Hamer PC, Robles SG, Zwinderman AH, Duffau $\mathrm{H}$, Berger MS: Impact of intraoperative stimulation brain mapping on glioma surgery outcome: a meta-analysis. J Clin Oncol 30:2559-2565, 2012

3. Duffau H, Lopes M, Arthuis F, Bitar A, Sichez JP, Van Effenterre R, et al: Contribution of intraoperative electrical stimulations in surgery of low grade gliomas: a comparative study between two series without (1985-96) and with (1996-2003) functional mapping in the same institution. J Neurol Neurosurg Psychiatry 76:845-851, 2005

4. Sanai N, Mirzadeh Z, Berger MS: Functional outcome after language mapping for glioma resection. $\mathbf{N}$ Engl J Med 358:18-27, 2008

5. Sanai N, Polley MY, McDermott MW, Parsa AT, Berger MS: An extent of resection threshold for newly diagnosed glioblastomas. J Neurosurg 115:3-8, 2011

6. Stummer W, Pichlmeier U, Meinel T, Wiestler OD, Zanella F, Reulen HJ: Fluorescence-guided surgery with 5-aminolevulinic acid for resection of malignant glioma: a randomised controlled multicentre phase III trial. Lancet Oncol 7:392-401, 2006

7. Szaflarski JP, Gloss D, Binder JR, Gaillard WD, Golby AJ, Holland SK, et al: Practice guideline summary: Use of fMRI in the presurgical evaluation of patients with epilepsy: Report of the Guideline Development, Dissemination, and Implementation Subcommittee of the American Academy of Neurology. Neurology 88:395-402, 2017 
Introduction: the rise of the robots in spinal surgery 